Artículo original

\title{
Costos médicos directos de las infecciones del tracto urinario por bacilos Gram negativos resistentes a betalactámicos en un hospital de alta complejidad de Medellín, Colombia
}

Carlos Andrés Vargas-Alzate ${ }^{1}$, Luis Felipe Hiquita-Gutiérrez ${ }^{1,2,3}$, Judy Natalia JiménezQuiceno'

${ }^{1}$ Línea de Epidemiología Molecular Bacteriana, Grupo de Microbiología Básica y Aplicada, Escuela de Microbiología, Universidad de Antioquia, Medellín, Colombia

${ }^{2}$ Grupo de Investigación en Salud y Sostenibilidad, Escuela de Microbiología, Universidad de Antioquia, Medellín, Colombia

${ }^{3}$ Grupo de Investigación INFETTARE, Facultad de Medicina, Universidad Cooperativa de Colombia, Medellín, Colombia

Institución donde se llevó a cabo el estudio:

Línea de Epidemiología Molecular Bacteriana, Grupo de Microbiología Molecular, Grupo de Investigación en Microbiología Básica y Aplicada (MICROBA), Escuela de Microbiología, Universidad de Antioquia, Medellín, Colombia

Introducción. Las infecciones del tracto urinario son muy frecuentes en el ámbito hospitalario. Debido a la aparición de la resistencia antimicrobiana, la complejidad de los procesos de atención ha aumentado y, con ello, la demanda de recursos.

Objetivo. Describir y comparar el exceso de los costos médicos directos de las infecciones del tracto urinario por Klebsiella pneumoniae, Enterobacter cloacae y Pseudomonas aeruginosa resistentes a betalactámicos.

Materiales y métodos. Se llevó a cabo un estudio de cohorte en una institución de tercer nivel de Medellín, Colombia, entre octubre del 2014 y septiembre del 2015. Se incluyeron los pacientes con infección urinaria, unos por bacterias sensibles a los antibióticos betalactámicos, y otros por bacterias resistentes a las cefalosporinas de tercera y cuarta generación y a los antibióticos carbapenémicos. Los costos se analizaron desde la perspectiva del sistema de salud. La información clínico-epidemiológica se obtuvo de las historias clínicas y los costos se calcularon utilizando los manuales tarifarios estándar. El exceso de costos se estimó mediante análisis multivariados.

Resultados. Se incluyeron 141 pacientes con infección urinaria: 55 (39\%) por bacterias sensibles a los betalactámicos, $54(38,3 \%)$ por bacterias resistentes a las cefalosporinas y $32(22,7 \%)$ por bacterias resistentes a los carbapenémicos. El exceso de costos totales ajustado de los 86 pacientes con infecciones del tracto urinario por bacterias resistentes a las cefalosporinas y a los carbapenémicos, fue de USD\$ $193\left(\right.$ IC $_{95 \%}-347$ a 734) y USD\$ $633\left(\mathrm{IC}_{95 \%}-50\right.$ a 1.316), respectivamente comparados con el grupo de 55 pacientes por bacterias sensibles a los betalactámicos. Las diferencias se presentaron principalmente en el uso de antibióticos de amplio espectro, como el meropenem, la colistina y la fosfomicina. Conclusión. Los resultados evidenciaron un incremento sustancial de los costos médicos directos de los pacientes con infecciones del tracto urinario por bacterias resistentes a las cefalosporinas o a los carbapenémicos. Esta situación genera especial preocupación en los países endémicos como Colombia, donde la alta frecuencia de infecciones del tracto urinario y de resistencia a los betalactámicos puede causar un mayor impacto económico en el sector de la salud.

Palabras claves: betalactámicos; resistencia bacteriana; costos de la atención en salud; Gram-negativos; infecciones del tracto urinario.

Direct medical costs of urinary tract infections by Gram-negative bacilli resistant to beta-lactams in a tertiary care hospital in Medellín, Colombia

Introduction: Urinary tract infections are very frequent in the hospital environment and given the emergence of antimicrobial resistance, they have made care processes more complex and have placed additional pressure on available healthcare resources.

Objective: To describe and compare excess direct medical costs of urinary tract infections due to Klebsiella pneumoniae, Enterobacter cloacae and Pseudomonas aeruginosa resistant to beta-lactams.

Materials and methods: A cohort study was conducted in a third level hospital in Medellín, Colombia, from October, 2014, to September, 2015. It included patients with urinary 
tract infections caused by beta-lactam-susceptible bacteria, third and fourth generation cephalosporin-resistant, as well as carbapenem-resistant. Costs were analyzed from the perspective of the health system. Clinical-epidemiological information was obtained from medical records and the costs were calculated using standard tariff manuals. Excess costs were estimated with multivariate analyses.

Results: We included 141 patients: 55 (39\%) were sensitive to beta-lactams, 54 (38.3\%) were resistant to cephalosporins and $32(22.7 \%)$ to carbapenems. The excess total adjusted costs of patients with urinary tract infections due to cephalosporin- and carbapenem-resistant bacteria were US\$193 (95\% confidence interval (CI): US\$ -347-734) and US\$ 633 (95\% Cl: US\$ -50-1316), respectively, compared to the group of patients with beta-lactam sensitive urinary tract infections. The differences were mainly found in the use of broad-spectrum antibiotics such as meropenem, colistin, and fosfomycin. Conclusion: Our results show a substantial increase in the direct medical costs of patients with urinary tract infections caused by beta-lactam-resistant Gram-negative bacilli (cephalosporins and carbapenems). This situation is of particular concern in endemic countries such as Colombia, where the high frequencies of urinary tract infections and the resistance to beta-lactam antibiotics can generate a greater economic impact on the health sector.

Keywords: Beta-lactams; bacterial resistance; health care costs; Gram-negative; urinary tract infections.

Después de las infecciones respiratorias, las urinarias son las más frecuentes en el ámbito hospitalario y en la comunidad, lo que motiva cerca de siete millones de consultas médicas ambulatorias y 100.000 hospitalizaciones anuales (1-3). Dichas infecciones se han asociado, principalmente, con el uso de dispositivos invasivos como los catéteres urinarios, así como con complicaciones como la bacteriemia y la septicemia $(4,5)$.

Entre los agentes etiológicos de las infecciones del tracto urinario, los bacilos Gram negativos resistentes a los betalactámicos constituyen un grave problema de salud pública por su alta frecuencia, y por su impacto clínico en términos de una mayor morbilidad y de prolongadas hospitalizaciones, además de la carga económica que implica su atención $(6,7)$.

A nivel mundial, se reporta con mucha frecuencia la presencia de bacterias resistentes a las cefalosporinas de tercera y cuarta generación: entre 20,8 y $26 \%$ en Europa $(8,9)$ entre 20,2 y $25,5 \%$ en África $(6,10)$ y hasta de 32,6\% en Latinoamérica (11). Por otra parte, en la última década, el uso intensivo de los carbapenémicos -considerados como la última opción terapéutica segura para el tratamiento de infecciones multirresistentes- ha generado la aparición y la diseminación de la resistencia a esta familia de antibióticos en todo el mundo, con frecuencias hasta de $19 \%$ en Estados Unidos (12) y de $22 \%$ en Europa $(7,13,14)$.

Tradicionalmente, se ha considerado que la atención hospitalaria de las infecciones del tracto urinario tiene un bajo costo, pero su alta frecuencia y el aumento de la prevalencia de bacterias resistentes a los betalactámicos, han acrecentado su impacto financiero en el sector salud. En los Estados Unidos, se ha estimado que los costos médicos anuales (atención ambulatoria, medicamentos y gastos de hospitalización) de estas infecciones pueden llegar a ser de USD\$ 474 millones, otros costos no médicos (transporte, días de enfermedad y morbilidad), hasta de USD\$185 millones, y los costos indirectos (pérdida de producción laboral), de USD $\$ 936$ millones $(15,16)$.

En Medellín, la segunda ciudad más importante de Colombia, se presenta una situación compleja en torno a la resistencia a los betalactámicos, ya que se han observado altos porcentajes de resistencia a las cefalosporinas en los últimos años, en las unidades de cuidados intensivos (19,4\%), así como en otros servicios hospitalarios $(27,9 \%)$ y en la atención ambulatoria (15\%) 
(17). Asimismo, en las unidades de cuidados intensivos, es muy frecuente la resistencia a los carbapenémicos, como imipenem en Klebsiella pneumoniae (5,7 \%) y en Pseudomonas aeruginosa (22,3\%), además de resistencia a meropenem (16,1\%) en Acinetobacter baumannii y a ertapenem (16,5\%) en Enterobacter cloacae (17).

Esta situación es preocupante, dado que en los estudios realizados previamente en la ciudad, las infecciones del tracto urinario han sido las más frecuentemente causadas por bacilos Gram negativos resistentes a los betalactámicos (18-20). A pesar de ello, no hay estudios locales que describan la carga económica que tal situación genera en el sector de la salud, y que respalden la adopción de decisiones sobre el uso de antibióticos y otras intervenciones en estos pacientes.

En este sentido, el objetivo del presente trabajo fue describir y comparar el exceso de los costos médicos directos de las infecciones del tracto urinario por K. pneumoniae, E. cloacae y $P$. aeruginosa resistentes a los betalactámicos en un hospital de alto nivel de complejidad de Medellín.

\section{Materiales y métodos}

\section{Diseño y población de estudio}

Se llevó a cabo un estudio de cohorte entre el $1^{\circ}$ de octubre de 2014 y el 30 de septiembre de 2015, en una institución de Medellín de alto nivel de complejidad con una capacidad de 754 camas. Se incluyeron todos los pacientes mayores de 18 años hospitalizados en los diferentes pabellones con diagnóstico de infección del tracto urinario por K. pneumoniae, $E$. cloacae o $P$. aeruginosa. No se excluyó a ningún paciente del estudio.

\section{Identificación y sensibilidad de los aislamientos bacterianos}

La identificación y la sensibilidad de los aislamientos se establecieron empleando el sistema VITEK $2^{\text {TM }}$ (bioMérieux, Marcy l'Étoile, France). Los controles de calidad y la interpretación de la sensibilidad se determinaron según las normas establecidas por el Clinical and Laboratory Standards Institute (CLSI), 2015 (21).

\section{Grupos de comparación}

A partir de la sensibilidad de los aislamientos, se conformaron tres grupos de comparación, como se explica a continuación.

Sensibles a betalactámicos (grupo no expuesto). Pacientes infectados por $K$. pneumoniae, E. cloacae o $P$. aeruginosa sensibles a los betalactámicos (cefalosporinas de tercera o cuarta generación y carbapenémicos).

Resistentes a cefalosporinas de tercera y cuarta generación (grupo expuesto 1). Pacientes infectados por K. pneumoniae, E. cloacae o P. aeruginosa resistentes, por lo menos, a una cefalosporina de tercera o cuarta generación.

Resistentes a carbapenémicos (grupo expuesto 2). Pacientes infectados por K. pneumoniae, E. cloacae o P. aeruginosa resistentes, por lo menos, a un carbapenémico ( $K$. pneumoniae y $E$. cloacae: a ertapenem, imipenem, meropenem y doripenem; $P$. aeruginosa: a imipenem, meropenem y doripenem).

El exceso de costos debido a la resistencia se definió como el costo 'incremental' (sic) de un paciente con infección urinaria por bacterias resistentes 
a cefalosporinas de tercera y cuarta generación o a carbapenémicos, frente a los costos de un paciente con infección urinaria por bacterias sensibles (22).

\section{Recolección de la información clínico-epidemiológica}

La información clínico-epidemiológica de cada paciente se obtuvo a partir de la revisión de las historias clínicas. Se incluyeron las características sociodemográficas, la descripción de la infección, las comorbilidades, la hospitalización y la evolución del paciente una vez finalizado el tratamiento antibiótico. Para medir la gravedad de las comorbilidades, se utilizó el índice de Charlson (23), en tanto que la clasificación de las infecciones asociadas a la atención en salud o adquiridas en la comunidad se ajustó a las definiciones de los Centers for Disease Control and Prevention (CDC) (24).

\section{Estimación de los costos médicos directos}

Las variables incluidas en la estimación de los costos directos se seleccionaron según los rubros considerados en estudios previos similares a este (25-28). Se conformó un panel integrado por un médico infectólogo, un epidemiólogo, un experto en resistencia bacteriana y un gerente de sistemas de información en salud. Además, se diseñó un formulario que incluyó todos los rubros correspondientes a costos totales, estancia hospitalaria, medicamentos (especificando la contribución de los antibióticos), pruebas de laboratorio, tratamiento antibiótico ambulatorio, procedimientos quirúrgicos, interconsultas e imágenes diagnósticas, y se estableció el costo de cada uno de ellos.

Se calcularon los costos día a día a partir del momento del diagnóstico clínico y hasta finalizado el tratamiento antibiótico desde la perspectiva del Sistema General de Seguridad Social en Salud de Colombia. El costo de los medicamentos se estableció con base en el reporte de los precios del 2014 en el sistema de medicamentos del Ministerio de Salud, en tanto que el costo de los procedimientos quirúrgicos, interconsultas, exámenes de laboratorio, imágenes diagnósticas y estancia hospitalaria, se tomó del manual tarifario del Instituto de Seguros Sociales (Acuerdo No. 256 de 2001), con un incremento del 30 \% según la recomendación del Instituto de Evaluación Tecnológica en Salud (29).

El costo total de los medicamentos se obtuvo multiplicando el número de dosis suministradas al paciente por el costo unitario del medicamento registrado en el manual de medicamentos. El costo total de los procedimientos se calculó multiplicando el precio del procedimiento según el manual de procedimientos por el número de veces que este se aplicó al paciente. En cuanto a los procedimientos quirúrgicos, el costo se calculó sumando los honorarios médicos del cirujano, del anestesiólogo, del ayudante quirúrgico, de los derechos de sala y de los materiales.

Los costos se determinaron en pesos colombianos y luego se convirtieron a dólares estadounidenses, teniendo en cuenta el promedio de la tasa de cambio del 2014 publicada por el Banco de la República de Colombia (30).

\section{Análisis estadístico}

Se utilizaron frecuencias absolutas y relativas en las variables cualitativas. Se determinó el cumplimiento del supuesto de normalidad con la prueba de Shapiro-Wilk y se calcularon las medianas con el rango intercuartílico en las variables cuantitativas. 
La diferencia entre las variables categóricas se determinó a partir de la prueba de ji al cuadrado de Pearson y, para las cuantitativas, se utilizó la prueba de Kruskal-Wallis y, posteriormente, una prueba $U$ de Mann-Whitney con corrección de Bonferroni, para conocer entre cuáles categorías se presentaban diferencias significativas (31).

Para los análisis bivariado y multivariado, se emplearon modelos lineales generalizados con distribución gamma y función de enlace de identidad para cada una de las variables costeadas $(\beta=0,75 ; \alpha=1,33)$. En el análisis multivariado, se tomó como resultado, o desenlace, el exceso de costos, el cual se ajustó por las variables que pudieran afectar la diferencia de los costos, como los días de hospitalización antes del diagnóstico de la infección, la bacteria aislada, las comorbilidades presentes en el momento del ingreso del paciente y la muerte durante la hospitalización, establecidas en el análisis bivariado, así como en estudios previos. Se consideraron significativos los valores de $p$ menores de 0,05 y todos los datos se procesaron en el programa Stata $^{\mathrm{TM}}$, versión 12.

\section{Consideraciones éticas}

El estudio fue aprobado por el Comité de Bioética para la Investigación en Humanos de la Sede de Investigación Universitaria de la Universidad de Antioquia (Acta 14-35-572). Se obtuvo, además, la aprobación del comité de ética del hospital participante.

\section{Resultados}

Se registraron 141 pacientes con infección del tracto urinario durante el periodo de estudio, de los cuales: $39(27,7 \%)$ habían tenido infecciones asociadas con la sonda vesical; 55 (39\%) habían presentado infecciones por bacterias sensibles a los betalactámicos; 54 (38,3\%) habían tenido infecciones por bacterias resistentes a las cefalosporinas de tercera y cuarta generación, y $32(22,7 \%)$ habían contraído infecciones por bacterias resistentes a los carbapenémicos (cuadro 1).

La mayoría de los pacientes eran hombres $(69,1 \%$ de los sensibles a betalactámicos, $63,0 \%$ de los resistentes a cefalosporinas y $84,4 \%$ de los resistentes a carbapenémicos). La mediana de edad fue similar: 72 años (rango intercuartílico, RIC=56-81); 70,5 años $(\mathrm{RIC}=51-80)$ y 70 años $(\mathrm{RIC}=58-79)$, respectivamente.

Las principales especialidades tratantes fueron Medicina Interna $(54,5 \%$, $46,3 \%$ y $40,6 \%$, respectivamente) y Urología $(21,8 \%, 24,1 \%$ y $31,3 \%$, respectivamente). El índice de Charlson fue similar en los tres grupos: 4 ( $\mathrm{RIC}=3-6), 4,5(\mathrm{RIC}=3-6)$ y 4 ( $\mathrm{RIC}=2,5-5,5)$ respectivamente. Las comorbilidades más frecuentes fueron diabetes $(32,7 \%, 27,8 \%$ y $18,8 \%$, respectivamente) y enfermedad pulmonar $(21,8 \%, 25,9 \%$ y $21,9 \%$, respectivamente).

En todos los grupos prevalecieron las infecciones adquiridas en la comunidad (70,9 \%, 75,9 \% y 59,4 \%, respectivamente) y K. pneumoniae fue la bacteria que se aisló con mayor frecuencia (63,6 \%, 94,4 \% y 53,1\%, respectivamente).

En el grupo con bacterias sensibles a los betalactámicos y en aquel con resistencia a los carbapenémicos, fue más frecuente la necesidad de mantener la hospitalización al finalizar el tratamiento antibiótico $(47,3 \%$ y $46,9 \%$, respectivamente), en tanto que en el grupo con resistencia a las cefalosporinas predominó la hospitalización en casa (40,7 \%). La mediana de 
días de estancia hospitalaria fue similar en los tres grupos: $8(R I C=5-11), 10$ $(\mathrm{RIC}=7-12)$ y10 $(\mathrm{RIC}=6,5-14)$, respectivamente.

No se encontraron diferencias significativas en las variables que podían determinar una diferencia en los costos entre los grupos, tales como la hospitalización previa a la infección y las comorbilidades (cuadro 1).

\section{Descripción de los costos según los grupos de pacientes}

La mediana de los costos totales de hospitalización en el grupo de pacientes infecciones del tracto urinario resistentes a las cefalosporinas, fue de USD\$1.236 (RIC=909-1.854) y, en el de resistentes a los carbapenémicos fue de USD\$1.473 (RIC: 916-2.944), en tanto que, en el grupo de sensibles a betalactámicos fue de USD $892(\mathrm{RIC}=601-1.577)$.

Cuadro 1. Características clínico-epidemiológicas de los pacientes con infecciones del tracto urinario por bacterias sensibles a los betalactámicos, resistentes a las cefalosporinas de tercera y cuarta generación, y a los carbapenémicos

\begin{tabular}{|c|c|c|c|c|}
\hline Variable & $\begin{array}{l}\text { No expuestos } \\
\text { ITU sensibles a } \\
\text { betalactámicos } \\
\quad(n=55)\end{array}$ & $\begin{array}{l}\text { Expuesto } 1 \\
\text { ITU resistentes a } \\
\text { cefalosporinas } \\
(n=54)\end{array}$ & $\begin{array}{l}\text { Expuesto } 2 \\
\text { ITU resistentes a } \\
\text { carbapenémicos } \\
(\mathrm{n}=32)\end{array}$ & $\mathbf{p}$ \\
\hline Edad [mediana (RIC)] & $72(56,0-81,0)$ & $70,5 \quad(51-80)$ & $70(58-79)$ & $0,841^{\dagger}$ \\
\hline Sexo, hombre $[\mathrm{n}(\%)]$ & $38(69,1)$ & $34(63,0)$ & $27(84,4)$ & $0,108^{*}$ \\
\hline $\begin{array}{l}\text { Días transcurridos entre el ingreso al hospital y } \\
\text { el diagnóstico clínico [mediana (RIC)] }\end{array}$ & $0(0-0)$ & $0(0-1)$ & $0(0-6)$ & $0,067^{\dagger}$ \\
\hline \multicolumn{5}{|l|}{ Especialidad tratante [n (\%)] } \\
\hline Medicina interna & $30(54,5)$ & $25(46,3)$ & $13(40,6)$ & $0,486^{*}$ \\
\hline Urología & $12(21,8)$ & $13(24,1)$ & $10(31,3)$ & \\
\hline Nefrología & $7(12,7)$ & $11(20,4)$ & $4(12,5)$ & \\
\hline Cirugía & $3(5,5)$ & $3(5,6)$ & $2(6,3)$ & \\
\hline Índice de Charlson [mediana $(\mathrm{RIC})^{\mathrm{a}}$ & $4(3-6)$ & $4,5 \quad(3-6)$ & $4 \quad(2,5-5,5)$ & $0,831^{\dagger}$ \\
\hline Infección asociada a la atención en salud [n (\%)] & $16(29,1)$ & $13(24,1)$ & $13(40,6)$ & $0,265^{\star}$ \\
\hline \multicolumn{5}{|l|}{ Bacteria aislada [n (\%)] } \\
\hline Klebsiella pneumoniae & $35(63,6)$ & $51(94,4)$ & $17(53,1)$ & $<0,001^{*}$ \\
\hline Pseudomonas aeruginosa & $11(20,0)$ & $3(5,6)$ & $6(18,8)$ & \\
\hline Enterobacter cloacae & $9(16,4)$ & - & $9(28,1)$ & \\
\hline ITU asociada con uso de sonda vesical [n (\%)] & $11(20,0)$ & $14(25,9)$ & $14(43,8)$ & $0,054^{*}$ \\
\hline \multicolumn{5}{|l|}{ Evolución [n (\%)] } \\
\hline Continuó hospitalizado & $26(47,3)$ & $16(29,6)$ & $15(46,9)$ & $<0,001^{*}$ \\
\hline Hospitalización en casac & $5 \quad(9,1)$ & $22(40,7)$ & $7(21,9)$ & \\
\hline Mejoría $^{d}$ & $18(32,7)$ & $6(11,1)$ & $3(9,4)$ & \\
\hline Muerte & $2(3,6)$ & 0 & $3 \quad(9,4)$ & \\
\hline $\begin{array}{l}\text { Días transcurridos entre la fecha del diagnóstico } \\
\text { clínico y la finalización del tratamiento antibiótico } \\
\text { [mediana }(\mathrm{RIC})]\end{array}$ & $8(5-11)$ & $10(7-12)$ & $10 \quad(6,5-14)$ & $0,159^{\dagger}$ \\
\hline
\end{tabular}

ITU: infecciones del tracto urinario; RIC: rango intercuartílico

En negrilla, $p<0,05$

* Prueba de ji al cuadrado de Pearson

† Prueba de Kruskal-Wallis

a Índice de comorbilidades para enfermedades crónicas que pueden alterar a corto plazo el riesgo de morir del paciente y que se presentan durante toda la hospitalización. Los puntajes de las enfermedades se encuentran entre 1 y 6 , y después de los 50 años el índice se incrementa en un punto por cada década. Cuanto más alto el puntaje, mayor es la gravedad de las comorbilidades.

${ }^{\mathrm{b}}$ El paciente finalizó el tratamiento antibiótico y siguió hospitalizado.

cPor su situación clínica, el paciente fue atendido con tratamiento antibiótico intravenoso en su lugar de residencia con los elementos y por el personal requerido para una adecuada recuperación.

d El paciente se recuperó parcialmente de la infección y continuó tomando antibióticos por vía oral después de ser dado de alta. 
Con respecto al grupo de infecciones sensibles a los betalactámicos, los antibióticos representaron la mayor diferencia en la mediana de los costos: en el grupo con resistencia a las cefalosporinas dicha mediana fue de USD\$ $103(p<0,001) y$, en aquel con resistencia a los carbapenémicos fue de USD\$ $102(p<0,001)$ (cuadro 2). Estos resultados siguieron siendo significativos tras aplicar la corrección de Bonferroni.

Cuadro 2. Descripción de los costos de atención hospitalaria de los pacientes con infección del tracto urinario por bacterias sensibles a los betalactámicos, resistentes a las cefalosporinas de tercera y cuarta generación, y a los carbapenémicos

\begin{tabular}{|c|c|c|c|c|c|}
\hline Costos & $\begin{array}{r}\text { No } \\
\text { ITU s } \\
\text { beta } \\
\text { Median }\end{array}$ & $\begin{array}{l}\text { expuestos } \\
\text { sensibles a } \\
\text { lactámicos } \\
(n=55) \\
\text { na, USD (RIC) }\end{array}$ & $\begin{array}{c}\text { Expuestos } 1 \\
\text { ITU resistentes a } \\
\text { cefalosporinas } \\
(n=54) \\
\text { Mediana, USD (RIC) }\end{array}$ & $\begin{array}{c}\text { Expuestos } 2 \\
\text { ITU resistentes a } \\
\text { carbapenémicos } \\
(n=32) \\
\text { Mediana, USD (RIC) }\end{array}$ & $\mathbf{p}$ \\
\hline Total atención hospitalaria & 892 & $(601-1.577)$ & $1.236(909-1.854)$ & $1.473(916-2.944)$ & $0,018^{\star+}$ \\
\hline Antibióticos & 20 & $(12-66)$ & $123 \quad(60-203)$ & $122(49-881)$ & $<0,001^{\star \dagger}$ \\
\hline Otros medicamentos & 90 & $(37-229)$ & $105 \quad(76-229)$ & $131 \quad(69-190)$ & $0,259^{*}$ \\
\hline Antibióticos después de la hospitalización & 0,917 & $(0,655-3)$ & $(3-133)$ & $102 \quad(13-202)$ & $<0,001^{\star \dagger}$ \\
\hline Interconsultas & 43 & $(22-65)$ & $43 \quad(22-65)$ & $43 \quad(22-76)$ & $0,481^{*}$ \\
\hline Exámenes de laboratorio & 206 & $(141-322)$ & $189(109-353)$ & $205(178-283)$ & $0,722^{*}$ \\
\hline Imágenes diagnósticas & 92 & $(39-212)$ & $71 \quad(33-177)$ & $103(23-269)$ & $0,716^{\star}$ \\
\hline Otros procedimientos & 62 & $(17-73)$ & $(6-99)$ & $(6-81)$ & $0,925^{*}$ \\
\hline Estancia hospitalaria (habitación) & 399 & $(228-761)$ & $513(342-733)$ & 518 (309-842) & $0,283^{*}$ \\
\hline
\end{tabular}

ITU: infecciones del tracto urinario; RIC: rango intercuartílico

En negrilla: $p<0,05$

* Prueba de Kruskal-Wallis

†Prueba de Kruskal-Wallis con corrección de Bonferroni

Exceso de costos de los pacientes con infecciones del tracto urinario por bacterias resistentes a las cefalosporinas o a los carbapenémicos

El exceso de costos, crudo y ajustado, de los pacientes con infecciones del tracto urinario por bacterias resistentes a las cefalosporinas o a los carbapenémicos, se presenta en el cuadro 3.

En el análisis bivariado, el mayor exceso de costos correspondió a los antibióticos administrados durante la hospitalización. Después de ajustar los costos por los días de hospitalización antes del diagnóstico de la infección, la bacteria aislada, el índice de Charlson y la muerte durante la hospitalización, el exceso de los costos totales de la atención hospitalaria en el grupo con resistencia a las cefalosporinas fue de USD $\$ 193\left(\mathrm{IC}_{95 \%}-347\right.$ a 734$)$ y, en aquel con resistencia a los carbapenémicos, fue de USD\$ $633\left(\mathrm{IC}_{95 \%}-50\right.$ a 1.316).

El principal exceso de costos en los dos grupos con resistencia a los betalactámicos, correspondió al de los antibióticos administrados durante la hospitalización. En aquel con resistencia a las cefalosporinas, el exceso ajustado fue de USD $\$ 97\left(\mathrm{IC}_{95 \%} 41-154\right)$ y solo un paciente recibió tratamiento combinado. En el grupo con resistencia los carbapenémicos, el exceso de costos fue de USD $\$ 509$ ( IC $\left._{95 \%} 250-768\right)$, en el cual, el 59,4 $\%$ de los pacientes recibieron tratamientos combinados con meropenem, polimixina, fosfomicina y colistina. Los costos de los antibióticos por día fueron superiores en el grupo con resistencia a los betalactámicos, en los cuales las mayores diferencias se presentaron entre los días 3 y 10 después del diagnóstico clínico (figura 1). 
Cuadro 3. Exceso de costos de la atención hospitalaria de los pacientes con infecciones del tracto urinario por bacterias resistentes a las cefalosporinas de tercera y cuarta generación, y a los carbapenémicos

Expuesto 1

\begin{tabular}{|c|c|c|c|c|c|c|c|c|c|c|}
\hline Costos & $\begin{array}{l}\text { Exceso de costos } \\
\text { crudo por ITU } \\
\text { resistente a } \\
\text { cefalosporinas } \\
\text { (coeficiente, IC }{ }_{95 \%} \text { ) }\end{array}$ & $\mathbf{p}$ & $\begin{array}{l}\text { Exceso de costos } \\
\text { ajustado por ITU } \\
\text { resistente a } \\
\text { cefalosporinasa } \\
\text { (coeficiente, IC }{ }_{95 \%} \text { ) }\end{array}$ & $\mathbf{p}$ & $\begin{array}{r}\text { Exce } \\
\text { cru } \\
r e \\
\text { carb } \\
\text { (coef }\end{array}$ & $\begin{array}{l}\text { so de costos } \\
\text { Ido por ITU } \\
\text { sistente a } \\
\text { apenémicos } \\
\text { iciente, IC } \text { ic }_{95 \%} \text { ) }\end{array}$ & $\mathbf{p}$ & $\begin{array}{r}\text { Exc } \\
a \\
\text { ITL } \\
\text { carb } \\
(\text { coe }\end{array}$ & $\begin{array}{l}\text { eso de costos } \\
\text { ustado por } \\
\text { resistente a } \\
\text { apenémicos a } \\
\text { ficiente, } \text { IC }_{95 \%} \text { ) }\end{array}$ & $\mathbf{p}$ \\
\hline $\begin{array}{l}\text { Total por atención } \\
\text { hospitalaria }\end{array}$ & $242(-254-738)$ & 0,339 & $193(-347-734)$ & 0,483 & 710 & $(0,686-1.418)$ & 0,050 & 633 & $(-50-1.316)$ & 0,069 \\
\hline Antibióticos & $95 \quad(39-150)$ & $<0,001$ & $(41-154)$ & $<0,001$ & 520 & $(259-782)$ & $<0,001$ & 509 & $(250-768)$ & $<0,001$ \\
\hline Otros medicamentos & $111(-101-323)$ & 0,305 & $140 \quad(-54-334)$ & 0,156 & -51 & $(-213-111)$ & 0,537 & -8 & $(-151-134)$ & 0,910 \\
\hline Interconsultas & $-4 \quad(-18-11)$ & 0,601 & $-9 \quad(-23-6)$ & 0,243 & 9 & $(-9-28)$ & 0,319 & 10 & $(-7-28)$ & 0,239 \\
\hline Exámenes de laboratorio & $6 \quad(-62-75)$ & 0,855 & $-4 \quad(-79-72)$ & 0,925 & $-0,792$ & $(-80-78)$ & 0,984 & -18 & $(-95-59)$ & 0,649 \\
\hline Imágenes diagnósticas & $-16 \quad(-75-44)$ & 0,603 & $-31(-100-39)$ & 0,388 & 44 & $(-46-134)$ & 0,334 & 44 & $(-50-39)$ & 0,356 \\
\hline Otros procedimientos & $7 \quad(-34-49)$ & 0,728 & $1 \quad(-33-36)$ & 0,933 & 19 & $(-32-71)$ & 0,464 & 43 & $(-6-92)$ & 0,085 \\
\hline $\begin{array}{l}\text { Estancia hospitalaria } \\
\text { (habitación) }\end{array}$ & $-23(-268-223)$ & 0,857 & $-57(-273-159)$ & 0,606 & 59 & $(-249-366)$ & 0,708 & 11 & $(-226-248)$ & 0,925 \\
\hline
\end{tabular}

ITU: infecciones del tracto urinario

En negrilla, $p<0,05$

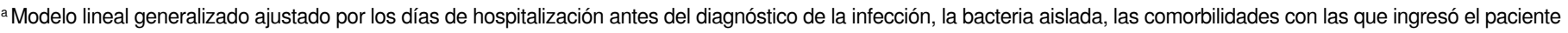
y la muerte durante la hospitalización

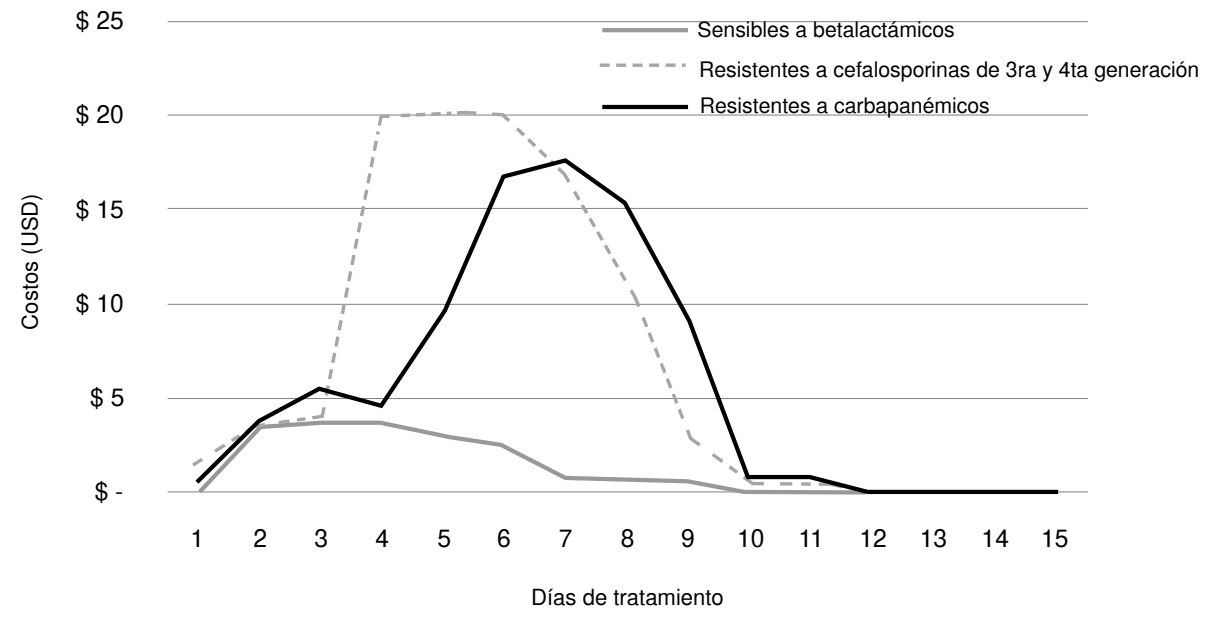

Figura 1. Distribución del exceso de los costos de antibióticos por día de los pacientes con infecciones del tracto urinario por bacterias sensibles a los betalactámicos, resistentes a las cefalosporinas de tercera y cuarta generación, y a los carbapenémicos

En cuanto a los antibióticos suministrados después de finalizar la hospitalización, se encontró que, en el grupo con resistencia a las cefalosporinas, la mediana de días de tratamiento fue de $6(\mathrm{RIC}=4-7)$ y prevaleció el uso del meropenem por vía intravenosa. En el grupo con resistencia a los carbapenémicos, dicha mediana fue de 8 ( $\mathrm{RIC}=5-10)$, y los antibióticos que se suministraron con mayor frecuencia fueron fosfomicina (un caso con fosfomicina más cefepime) y meropenem (dos casos con meropenem más polimixina $B$ y otro con meropenem más fosfomicina), ambos por vía intravenosa. 
Con respecto a las interconsultas, los exámenes de laboratorio, las imágenes diagnósticas, otros procedimientos y la estancia hospitalaria, los grupos con resistencia a los betalactámicos no presentaron excesos importantes en los costos, en comparación con el grupo con bacterias sensibles a los betalactámicos. Sin embargo, en el grupo con resistencia a las cefalosporinas, la frecuencia de las interconsultas por infectología fue de 10,2 $\%$ y, en aquel con resistencia a los carbapenémicos, fue de 65,6\%. Además, en ambos grupos con resistencia a los betalactámicos, se encontró una alta frecuencia de pruebas diagnósticas especializadas, como ultrasonografía de vías urinarias (26,5 y 40,6\%, respectivamente), tomografía computadorizada de abdomen y pelvis ( 4 y $15,6 \%$, respectivamente) y otros procedimientos como la inserción de catéter en la uretra (14,8 y 9,4\%, respectivamente).

Por último, en cuanto a la estancia hospitalaria y el tipo de habitación, el $2 \%$ del grupo con resistencia a las cefalosporinas estuvo cuatro días 0 más en las salas de aislamiento y, el $24,5 \%$, en salas individuales durante ocho días o más; del grupo con resistencia a los carbapenémicos, el 43,8 \% permaneció cuatro días o más en las salas de aislamiento y, el 21,9\%, en salas individuales ocho días o más.

\section{Discusión}

En este estudio, se pudo establecer que el costo total 'incremental' (sic) de las infecciones del tracto urinario por bacterias resistentes a cefalosporinas de tercera y cuarta generación fue de USD $\$ 193\left(\mathrm{IC}_{95 \%}-347\right.$ a 734) y por bacterias resistentes a carbapenémicos fue de USD $\$ 633\left(\right.$ IC $_{95 \%}$ -50 a 1.316) en comparación con los costos por infecciones por bacterias sensibles a los betalactámicos.

Entre los costos hospitalarios considerados, la mayor diferencia se presentó con los antibióticos, los cuales generaron excesos de costos de USD \$ $97\left(\mathrm{IC}_{95 \%} 41-154\right)$ en el grupo de resistentes a las cefalosporinas y de USD\$ 509 (IC ${ }_{95 \%}$ 250-768) en el grupo de resistentes a los carbapenémicos.

Aunque estos resultados evidencian la considerable carga económica generada por la resistencia bacteriana en el hospital de estudio, no pueden generalizarse a otros grupos poblacionales debido a las particularidades epidemiológicas de persona, tiempo y lugar, y a las del manejo de las infecciones en los hospitales. De ahí la importancia de llevar a cabo estudios de este tipo para conocer y comparar la carga de la resistencia bacteriana en dichas instituciones.

Considerando que las infecciones del tracto urinario se cuentan entre las más frecuentes en el ámbito hospitalario y comunitario, el impacto económico que puede generar la resistencia a cefalosporinas y a carbapenémicos representa un grave problema para el sistema de salud de los países endémicos como Colombia. Por ello, ante la creciente complejidad de los tratamientos y la demanda de recursos sanitarios, cada vez son más necesarios los estudios que entreguen información comprobada para implementar estrategias encaminadas a aumentar la calidad de la atención y disminuir los costos hospitalarios $(6,7)$.

Aunque en el mundo las infecciones del tracto urinario son de gran importancia por sus implicaciones clínicas y su alta prevalencia $(1,4,15)$, a nivel local la situación es alarmante. En estudios previos en Medellín, se encontró que dichas infecciones están entre las más frecuentes causadas por bacilos Gram negativos resistentes a los betalactámicos, con porcentajes 
de resistencia a los carbapenémicos de hasta $33,3 \%(18,20)$ y de resistencia a las cefalosporinas de hasta $47,6 \%(32,33)$, situación que genera un mayor impacto económico para las instituciones de salud y la comunidad, no solo por los costos hospitalarios (antibióticos, estancia hospitalaria, procedimientos quirúrgicos, entre otros), sino también por los costos sociales (pérdida de fuerza laboral y calidad de vida).

Actualmente, en algunos países de África y Latinoamérica, la frecuencia de infecciones del tracto urinario causadas por bacterias resistentes a las cefalosporinas alcanza el 25,5 y el $32,6 \%$, respectivamente $(6,11)$. Asimismo, las causadas por bacterias resistentes a los carbapenémicos alcanzan hasta el $10,7 \%$ en Corea (34), el $19 \%$ en los Estados Unidos (12) y el $22 \%$ en España (35), lo que demuestra que se trata de una problemática mundial.

La frecuencia de las infecciones del tracto urinario en la comunidad es cada vez más preocupante debido al aumento de bacilos Gram negativos resistentes a las cefalosporinas que, como ejemplos, llega hasta el 10,3\% en España (36) a $30 \%$ en Latinoamérica (37) y a $40 \%$ en Italia (38) en tanto que las infecciones por bacterias resistentes a los carbapenémicos alcanzan el $13 \%$ en Italia (38) y el $51,4 \%$ en Taiwán (39), lo que supone una gran carga financiera para los sistemas de salud (40).

Al comparar los costos 'incrementales' (sic) encontrados en este estudio con los de otros similares, se evidenció que, en el grupo con infecciones por bacterias resistentes a las cefalosporinas, el exceso fue menor a lo reportado en países como Estados Unidos (entre USD \$ 3.431 y USD\$ 16.451) $(25,26,41)$ y España (€\$1.109) (27).

Además de las diferencias propias de cada país en términos sociales, políticos y económicos, las discrepancias metodológicas tienen un papel muy importante en estos estudios, dado que dificultan la comparación directa de los resultados obtenidos. Entre las principales diferencias metodológicas, se cuenta la conformación de los grupos de comparación (resistentes a cefalosporinas $V s$. sensibles a cefalosporinas, infectados $V s$. no infectados), la inclusión de distintas especies bacterianas (Escherichia coli, K. pneumoniae y $P$. aeruginosa) y las mediciones de los costos hospitalarios relacionados con el proceso de atención (algunos estudios incluyen estancia, medicamentos y laboratorio, y otros, solamente los costos totales de la hospitalización).

En cuanto al análisis de los costos, se encontró que la mayoría de los estudios se hicieron desde la perspectiva institucional. Las diferencias metodológicas en los estudios de costos se han convertido en una limitación para la comparación de los resultados que apoyen la adopción de decisiones, por lo cual se sugiere el diseño de consensos metodológicos que permitan obtener resultados comparables entre instituciones de salud de diferentes países.

En cuanto al exceso de costos, es poca la información disponible en torno a pacientes con infecciones causadas por bacilos Gram negativos resistentes a los carbapenémicos. El exceso de USD\$ $633\left(\mathrm{IC}_{95 \%}-50\right.$ a 1.316) obtenido en este estudio, refleja la carga económica que la resistencia a los carbapenémicos genera, sobre todo en ciudades endémicas como Medellín. Establecer las causas que contribuyen a la resistencia bacteriana y su impacto económico es importante para implementar medidas de prevención y control de las infecciones por agentes patógenos resistentes, y para optimizar el uso de los recursos de salud a corto, a mediano y a largo plazo $(6,7)$. 
En el presente estudio, los costos de las infecciones del tracto urinario por bacterias resistentes a las cefalosporinas o resistentes a los carbapenémicos fueron mayores que los de los pacientes con infecciones por bacterias sensibles a los betalactámicos, principalmente en cuanto al uso de antibióticos de amplio espectro como el meropenem, la fosfomicina y la colistina. Asimismo, se evidenció que solo el 3,7 \% de los pacientes del grupo con resistencia a las cefalosporinas recibió tratamiento combinado con dos antibióticos, en tanto que el $37,5 \%$ del grupo con resistencia a los a los carbapenémicos recibió tratamientos combinados con dos antibióticos, el $18,8 \%$ con tres antibióticos y el 3,1 \% con cuatro antibióticos.

Entre las causas que pueden explicar el aumento de los costos hospitalarios, se encuentra la frecuencia con que se administran los antibióticos de amplio espectro como el meropenem y la colistina, considerados por el Ministerio de Salud colombiano como medicamentos de alto costo en el mercado nacional en comparación con otros países.

Esta particularidad, sumada a los altos costos de otros medicamentos, llevó a la conformación de una comisión nacional de precios de medicamentos cuyo principal objetivo es salvaguardar la sostenibilidad del Sistema General de Seguridad Social en Salud (42). Actualmente, la lista de antibióticos regulados incluye el meropenem, la colistina y el linezolid, y aunque en este estudio se encontró que la fosfomicina se usaba con gran frecuencia, su costo (USD\$102) es menor que el de otros antibióticos de amplio espectro no regulados por el Ministerio de Salud, como la tigeciclina (USD\$ 556) y el imipenem (USD\$ 525) (42). El uso frecuente de este tipo de antibióticos evidencia las dificultades del tratamiento médico cuando las infecciones del tracto urinario son causadas por bacterias resistentes.

Otro hallazgo destacado en este estudio fue que la mayoría de las infecciones resistentes a las cefalosporinas o a los carbapenémicos se presentó en el momento de la hospitalización (59,4 y 75,5\%, respectivamente), lo cual evidencia la importancia que han cobrado estas infecciones en el ámbito comunitario (43) además, la importancia de llevar a cabo estudios que trasciendan el ámbito hospitalario y detecten posibles fuentes de infección, determinen la frecuencia de colonización por bacterias resistentes en la comunidad y describan las costumbres relacionadas con el uso de los antibióticos.

En el presente estudio también se observó que, después de su egreso, muchos de los pacientes con infecciones del tracto urinario resistentes a las cefalosporinas o a los carbapenémicos continuaron el tratamiento antibiótico por vía intravenosa en su lugar de residencia (44,9 y 21,9\%, respectivamente). Varios autores han mencionado que este programa reduce los costos de hospitalización, y tiene ventajas sociales y familiares (mayor intimidad y comodidad del paciente, integración de la familia en el proceso de curación), sanitarias (reducción de riesgos relacionados con la estancia hospitalaria) y de gestión (utilización de las camas hospitalarias). En algunos de los estudios se ha asociado dicha estrategia con una disminución de hasta $38 \%$ de la mortalidad (44-47) Sin embargo, no existe información sobre el riesgo y las implicaciones que genera las medidas de hospitalización en casa de los pacientes con infecciones del tracto urinario por bacterias resistentes a las cefalosporinas o a los carbapenémicos, por lo cual es importante hacer estudios de costo-efectividad o costo-beneficio que determinen el impacto de esta intervención para las instituciones de salud y los convivientes (48-51). 
Por último, se ha mencionado la importancia de las interconsultas de infectología en los hospitales, principalmente en la mejoría de la calidad de la prescripción y en el uso de los antibióticos (52-56). Debido a la complejidad del manejo de las infecciones causadas por microorganismos resistentes a las cefalosporinas o a los carbapenémicos, en este estudio, la frecuencia de interconsultas por infectología fue del 10,2 y 65,6 \%, respectivamente. Aunque el número de interconsultas no fue significativamente alto, es importante resaltar que sus beneficios en términos de eficacia pueden ser mayores que los costos de la atención por parte de este tipo de profesionales en los centros asistenciales.

Una de las principales limitaciones de este estudio, y en general de los estudios de costos, es la ausencia de evaluaciones desde una perspectiva social que incluyan, no solo los costos relacionados con la atención hospitalaria, sino los gastos menores para el paciente e, incluso, la pérdida de fuerza laboral, relacionados directa o indirectamente con la infección $(48,57)$.

Otra limitación del estudio fue que no se incluyeron infecciones del tracto urinario causadas por $E$. coli, uno de los principales agentes patógenos causante de estas infecciones, ya que el propósito del estudio era la estimación del impacto económico de las infecciones del tracto urinario causadas por bacilos Gram negativos resistentes a las cefalosporinas o a los carbapenémicos y en $E$. coli la resistencia a carbapenémicos es muy baja. Además, se recolectó información de una sola institución de salud, por lo cual estos resultados no pueden extrapolarse a otras.

En conclusión, los pacientes con infecciones del tracto urinario por bacterias resistentes a las cefalosporinas o a los carbapenémicos generaron mayores costos totales de hospitalización que aquellos infectados por bacterias sensibles a los betalactámicos, principalmente, por los antibióticos. La alta frecuencia de estas infecciones en el ámbito hospitalario y comunitario, sumada a la resistencia a los betalactámicos, aumenta los costos de la atención en salud y exige la implementación de estrategias que permitan disminuir los altos porcentajes de resistencia, como son las políticas de uso de antibióticos y el fortalecimiento de los programas de vigilancia.

\section{Referencias}

1. Flores-Mireles AL, Walker JN, Caparon M, Hultgren SJ. Urinary tract infections: Epidemiology, mechanisms of infection and treatment options. Nat Rev Microbiol. 2015;13:269-84. https://doi.org/10.1038/nrmicro343

2. François M, Hanslik T, Dervaux B, Le Strat $Y$, Souty C, Vaux S, et al. The economic burden of urinary tract infections in women visiting general practices in France: A cross-sectional survey. BMC Health Serv Res. 2016;16:365. https://doi.org/10.1186/s12913-016-1620-2

3. Dielubanza EJ, Schaeffer AJ. Urinary tract infections in women. Med Clin North Am. 2011;95:27-41. https://doi.org/10.1016/j.mcna.2010.08.023

4. Pigrau C. Infecciones del tracto urinario nosocomiales. Enferm Infecc Microbiol Clin. 2013;31:614-24. https://doi.org10.1016/j.eimc.2012.11.015

5. Pavanello Silva C, Frota Mendonça SH, Romero Aquino C, Soares da Silva AF, Malacchia $\mathrm{JL}$, Campos Canesin A, et al. Principales factores de riesgo de infección del tracto urinario (ITU) en pacientes hospitalizados: propuesta de mejoras. Enfermería Global. 2009. Disponible en: http://scielo.isciii.es/scielo.php?script=sci arttext\&pid=S1695-61412009000100

6. El Bouamri MC, Arsalane L, El Kamouni Y, Zouhair S. Antimicrobial susceptibility of urinary Klebsiella pneumoniae and the emergence of carbapenem-resistant strains: A retrospective study from a university hospital in Morocco, North Africa. Afr J Urol. 2015;21:36-40. https://doi.org/10.1016/j.afju.2014.10.004 
7. Zowawi HM, Harris PN, Roberts MJ, Tambyah PA, Schembri MA, Pezzani MD, et al. The emerging threat of multidrug-resistant Gram-negative bacteria in urology. Nat Rev Urol. 2015;12:570-84. https://doi.org/10.1038/nrurol.2015.199

8. Romero ED, Padilla TP, Hernández AH, Grande RP, Vázquez MF, García IG, et al. Prevalence of clinical isolates of Escherichia coli and Klebsiella spp. producing multiple extended-spectrum $\beta$-lactamases. Diagn Microbiol Infect Dis. 2007;59:433-7. https://doi.org/10.1016/j.diagmicrobio.2007.06.007

9. Belmonte O, Drouet D, Alba J, Moiton MP, Kuli B, Lugagne-Delpon N, et al. Evolution of Enterobacteriaceae resistance to antibiotics in Reunion Island: emergence of extendedspectrum beta-lactamases. Pathol Biol (Paris). 2010;58:18-24. https://doi.org/10.1016/j.patbio.2009.07.021

10. Ben Haj Khalifa A, Khedher M. Epidemiological study of Klebsiella spp. uropathogenic strains producing extended-spectrum $\beta$-lactamase in a Tunisian university hospital, 2009. Pathol Biol (Paris). 2012;60:e1-5. https://doi.org/10.1016/j.patbio.2010.11.003

11. Villegas MV, Correa A, Pérez F, Miranda MC, Zuluaga T, Quinn JP. Prevalence and characterization of extended-spectrum $\beta$-lactamases in Klebsiella pneumoniae and Escherichia coli isolates from Colombian hospitals. Diagn Microbiol Infect Dis. 2004;49:21722. https://doi.org/10.1016/j.diagmicrobio.2004.03.001

12. Khawcharoenporn T, Vasoo S, Singh K. Urinary tract infections due to multidrug-resistant Enterobacteriaceae: Prevalence and risk factors in a Chicago emergency department. Emerg Med Int. 2013;2013:e258517. https://doi.org/10.1155/2013/258517

13. Paterson DL, Bonomo RA. Extended-spectrum beta-lactamases: A clinical update. Clin Microbiol Rev. 2005;18:657-86. https://doi.org/10.1128/CMR.18.4.657-686.2005

14. World Health Organization. Antimicrobial resistance: Global report on surveillance 2014. Fecha de consulta: 28 de septiembre de 2015. Disponible en: http://www.who.int/drugresistance/documents/surveillancereport/en/

15. Foxman B. Epidemiology of urinary tract infections: Incidence, morbidity, and economic costs. Am J Med. 2002;113(Suppl.1A):5S-13S.

16. Stone PW, Braccia D, Larson E. Systematic review of economic analyses of health careassociated infections. Am J Infect Control. 2005;33:501-9. https://doi.org/10.1016/S0002-9343(02)01054-9

17. Grupo para el Estudio de la Resistencia a Antibióticos de Medellín. Microorganismos. Fecha de consulta: 28 de septiembre de 2015. Disponible en: http://www.grupogermen.org/microorganismos.html

18. Vanegas JM, Cienfuegos AV, Ocampo AM, López L, del Corral H, Roncancio G, et al. Similar frequencies of Pseudomonas aeruginosa isolates producing KPC and VIM carbapenemases in diverse genetic clones at tertiary-care hospitals in Medellín, Colombia. J Clin Microbiol. 2014;52:3978-86. https://doi.org/10.1128/JCM.01879-14

19. Vanegas JM, Parra OL, Jiménez JN. Molecular epidemiology of carbapenem resistant Gram-negative bacilli from infected pediatric population in tertiary-care hospitals in Medellín, Colombia: An increasing problem. BMC Infect Dis. 2016;16:463. https://doi.org/10.1186/s12879-016-1805-7

20. Ocampo AM, Chen L, Cienfuegos AV, Roncancio G, Chavda KD, Kreiswirth BN, et al. A two-year surveillance in five Colombian tertiary care hospitals reveals high frequency of non-CG258 clones of carbapenem-resistant Klebsiella pneumoniae with distinct clinical characteristics. Antimicrob Agents Chemother. 2015;60:332-42. https://doi.org/10.1128/AAC.01775-15

21. Clinical and Laboratory Standards Institute. Performance standards for antimicrobial susceptibility testing: Twenty-fifth informational supplement. CLSI document M100-S25. Wayne, PA: Clinical and Laboratory Standards Institute; 2015.

22. Tansarli GS, Karageorgopoulos DE, Kapaskelis A, Falagas ME. Impact of antimicrobial multidrug resistance on inpatient care cost: An evaluation of the evidence. Expert Rev Anti Infect Ther. 2013;11:321-31. https://doi.org/10.1586/eri.13.4

23. Charlson ME, Pompei P, Ales KL, MacKenzie CR. A new method of classifying prognostic comorbidity in longitudinal studies: Development and validation. J Chronic Dis. 1987;40:373-83.

24. Centers for Disease Control and Prevention. Identifying healthcare-associated infections (HAl) for NHSN surveillance. Fecha de consulta: 9 de enero de 2015. Disponible en: http://www.cdc.gov/nhsn/PDFs/pscManual/2PSC IdentifyingHAls NHSNcurrent.pdf 
25. MacVane SH, Tuttle LO, Nicolau DP. Impact of extended-spectrum $\beta$-lactamase-producing organisms on clinical and economic outcomes in patients with urinary tract infection. J Hosp Med. 2014;9:232-8. https://doi.org/10.1002/jhm.2157

26. Lee SY, Kotapati S, Kuti JL, Nightingale CH, Nicolau DP. Impact of extended-spectrum beta-lactamase-producing Escherichia coli and Klebsiella species on clinical outcomes and hospital costs: A matched cohort study. Infect Control Hosp Epidemiol. 2006;27:1226-32. https://doi.org/10.1086/507962

27. Esteve-Palau E, Solande G, Sánchez F, Sorlí L, Montero M, Güerri R, et al. Clinical and economic impact of urinary tract infections caused by ESBL-producing Escherichia coli requiring hospitalization: A matched cohort study. J Infect. 2015;71:667-74. https://doi.org/10.1016/j.jinf.2015.08.012

28. Cardwell SM, Crandon JL, Nicolau DP, McClure MH, Nailor MD. Epidemiology and economics of adult patients hospitalized with urinary tract infections. Hosp Pract (1995). 2016;44:33-40. https://doi.org/10.1080/21548331.2016.1133214

29. Instituto de Evaluación Tecnológica en Salud. Manual para la elaboración de evaluaciones económicas en salud. Fecha de consulta: 28 de septiembre de 2015. Disponible en: http://www.iets.org.co/manuales

30. Banco de la República. Tasa de cambio del peso colombiano (TRM). Fecha de consulta: 28 de septiembre de 2016. Disponible en: http://www.banrep.gov.co/es/trm

31. Donado-Campos JM, Rodríguez-Arango GO. Epidemiología y metodología científica aplicada a la pediatría (III): introducción al análisis estadístico en epidemiología. An Esp Pediatr. 1999;50:81-90.

32. Jiménez-Jiménez J, Jiménez JG, Barrera ME, Arias JK, Velilla DM, Marín AE, et al. Caracterización clínica, microbiológica y de sensibilidad a antimicrobianos en pacientes con infección nosocomial del tracto urinario: cuatro años y medio de vigilancia epidemiológica. Med UNAB. 2012;14.

33. Orrego-Marín CP, Henao-Mejía CP, Cardona-Arias JA. Prevalencia de infección urinaria, uropatógenos y perfil de susceptibilidad antimicrobiana. Acta Médica Colombiana. 2014;39:352-8.

34. Song JY, Cheong HJ, Choi WS, Heo JY, Noh JY, Kim WJ. Clinical and microbiological characterization of carbapenem-resistant Acinetobacter baumannii bloodstream infections. J Med Microbiol. 2011;60:605-11. https://doi.org/10.1099/jmm.0.029439-0

35. Álvarez-Otero J, Lamas-Ferreiro JL, González-González L, Rodríguez-Conde I, FernándezSoneira MJ, Arca-Blanco A, et al. Carbapenem resistance in Pseudomonas aeruginosa isolated from urine cultures: Prevalence and risk factors. Rev Espanola Quimioter. 2017;30:183-94.

36. Boix-Palop L, Xercavins M, Badía C, Obradors M, Riera M, Freixas N, et al. Emerging extended-spectrum $\beta$-lactamase-producing Klebsiella pneumoniae causing communityonset urinary tract infections: A case-control-control study. Int J Antimicrob Agents. 2017;50:197-202. https://doi.org/10.1016/j.ijantimicag.2017.03.009

37. Bours PHA, Polak R, Hoepelman AIM, Delgado E, Jarquin A, Matute AJ. Increasing resistance in community-acquired urinary tract infections in Latin America, five years after the implementation of national therapeutic guidelines. Int J Infect Dis. 2010;14:e770-4. https://doi.org/10.1016/j.ijid.2010.02.2264

38. Salerno F, Borzio M, Pedicino C, Simonetti R, Rossini A, Boccia S, et al. The impact of infection by multidrug-resistant agents in patients with cirrhosis. A multicenter prospective study. Liver Int. 2017;37:71-9. https://doi.org/10.1111/liv.13195

39. Zilberberg MD, Nathanson BH, Sulham K, Fan W, Shorr AF. Carbapenem resistance, inappropriate empiric treatment and outcomes among patients hospitalized with Enterobacteriaceae urinary tract infection, pneumonia and sepsis. BMC Infect Dis. 2017;17:279. https://doi.org/10.1186/s12879-017-2383-z

40. Brizendine KD, Richter SS, Cober ED, van Duin D. Carbapenem-resistant Klebsiella pneumoniae urinary tract infection following solid organ transplantation. Antimicrob Agents Chemother. 2015;59:553-7. https://doi.org/10.1128/AAC.04284-14

41. Cardwell SM, Crandon JL, Nicolau DP, McClure MH, Nailor MD. Epidemiology and economics of adult patients hospitalized with urinary tract infections. Hosp Pract 1995. 2016;44:33-40. https://doi.org/10.1080/21548331.2016.1133214

42. Ministerio de Salud y Protección Social. Regulación de precios de medicamentos, 2015. Fecha de consulta: 6 de febrero de 2017. Disponible en: https://www.minsalud.gov.co/salud/MT/Paginas/medicamentos-regulacion-precios.aspx 
43. Hoyos Á, Serna L, Ortiz G, Aguirre J. Infección urinaria adquirida en la comunidad en pacientes pediátricos: clínica, factores de riesgo, etiología, resistencia a los antibióticos y respuesta a la terapia empírica. Infectio. 2012;16:94-103. https://doi.org/10.1016/S0123-9392(12)70062-0

44. González-Ramallo VJ, Valdivieso-Martínez B, Ruiz-García V. Hospitalización a domicilio. Med Clín(Barc). 2002;118:659-64. https://doi.org/10.1016/S0025-7753(02)72488-3

45. Alonso G, Escudero JM. La unidad de corta estancia de urgencias y la hospitalización a domicilio como alternativas a la hospitalización convencional. An Sist Sanit Navar. 2010;33:97-106.

46. Cryer L, Shannon SB, Amsterdam MV, Leff B. Costs for 'hospital at home' patients were 19 percent lower, with equal or better outcomes compared to similar inpatients. Health Aff (Millwood). 2012;31:1237-43. https://doi.org/10.1377/hlthaff.2011.1132

47. Shepperd S, Doll H, Angus RM, Clarke MJ, Iliffe S, Kalra L, et al. Avoiding hospital admission through provision of hospital care at home: A systematic review and meta-analysis of individual patient data. CMAJ. 2009;180:175-82. https://doi.org/10.1503/cmaj.081491

48. Cohen B, Larson EL, Stone PW, Neidell M, Glied SA. Factors associated with variation in estimates of the cost of resistant infections. Med Care. 2010;48:767-75. https://doi.org/10.1097/MLR.0b013e3181e358b9

49. García-Altés A, Navas E, Soriano MJ. Evaluación económica de intervenciones de salud pública. Gac Sanit. 2011;25:25-31. https://doi.org/10.1016/S0213-9111(11)70005-X

50. Coast J, Smith R, Karcher A-M, Wilton P, Millar M. Superbugs II: How should economic evaluation be conducted for interventions which aim to contain antimicrobial resistance? Health Econ. 2002;11:637-47.

51. Shang J, Ma C, Poghosyan L, Dowding D, Stone P. The prevalence of infections and patient risk factors in home health care: A systematic review. Am J Infect Control. 2014;42:479-84. https://doi.org/10.1016/j.ajic.2013.12.018

52. Cisneros JM, Palomino-Nicás J, Pachón-Díaz J. La interconsulta de enfermedades infecciosas es una actividad clave para los servicios y unidades de enfermedades infecciosas y para el hospital. Enferm Infecc Microbiol Clin. 2014;32:671-5. https://doi.org/10.1016/j.eimc.2013.03.019

53. Lo E, Rezai K, Evans AT, Madariaga MG, Phillips M, Brobbey W, et al. Why don't they listen? Adherence to recommendations of infectious disease consultations. Clin Infect Dis. 2004;38:1212-8. https://doi.org/10.1086/383315

54. Rodríguez-Baño J, Paño-Pardo JR, Álvarez-Rocha L, Asensio Á, Calbo E, Cercenado E, et al. Programas de optimización de uso de antimicrobianos (PROA) en hospitales españoles: documento de consenso GEIH-SEIMC, SEFH y SEMPSPH. Enferm Infecc Microbiol Clin. 2012;30:e1-22.e23. https://doi.org/10.1016/j.eimc.2011.09.018

55. Pulcini C, Botelho-Nevers E, Dyar OJ, Harbarth S. The impact of infectious disease specialists on antibiotic prescribing in hospitals. Clin Microbiol Infect. 2014;20:963-72. https://doi.org/10.1111/1469-0691.12751

56. Cassenote AJ, Scheffer MC, Segurado AA, Cassenote AJ, Scheffer MC, Segurado AA. Brazilian infectious diseases specialists: Who and where are they? Braz J Infect Dis. 2016;20:141-8. https://doi.org/10.1016/j.bjid.2015.10.009

57. Gandra S, Barter DM, Laxminarayan R. Economic burden of antibiotic resistance: How much do we really know? Clin Microbiol Infect. 2014;20:973-80. https://doi.org/10.1111/1469-0691.12798 